\title{
Conditional probability and defeasible inference ${ }^{\dagger}$
}

\author{
Horacio Arló Costa (hcosta@andrew.cmu.edu) \\ Carnegie Mellon University \\ Philosophy Department \\ Pittsburgh, P.A. 15213 \\ Rohit Parikh (ripbc@cunyvm.cuny.edu) \\ $C U N Y$ and $N Y U$ \\ CUNY Graduate Center \\ Department of Computer Science \\ 365 Fifth Avenue \\ New York NY 10016-4309
}

\begin{abstract}
We offer a probabilistic model of rational consequence relations (Lehmann and Magidor, 1990) by appealing to the extension of the classical Ramsey-Adams test proposed by Vann McGee in (McGee, 1994). Previous and influential models of non-monotonic consequence relations have been produced in terms of the dynamics of expectations (Gärdenfors and Makinson, 1994), (Gärdenfors, 1993). 'Expectation' is a term of art in these models, which should not be confused with the notion of expected utility. The expectations of an agent are some form of belief weaker than absolute certainty. Our model offers a modified and extended version of an account of qualitative belief in terms of conditional probability, first presented in (van Fraassen, 1995). We use this model to relate probabilistic and qualitative models of nonmonotonic relations in terms of expectations. In doing so we propose a probabilistic model of the notion of expectation.

We provide characterization results both for logically finite languages and for logically infinite, but countable, languages. The latter case shows the relevance of the axiom of countable additivity for our probability functions. We show that a rational logic defined over a logically infinite language can only be fully characterized in terms of finitely additive conditional probability.
\end{abstract}

Keywords: Belief Revision, Conditional Probability, Conditionals, Countable Additivity, Non-Monotonic Logic

\section{Introduction}

Various methods of inference used in scientific and computational reasoning violate the law of monotonicity. This law stipulates that whenever a conclusion is inferred from a given set of premises, the same conclusion is also a consequence of that set of premises augmented by additional information. Dov Gabbay first proposed to study these forms of defeasible reasoning through properties of its associated consequence

$\dagger$ The research of both authors was supported in part by a grant from NSF, and, for Parikh, also by support from the research foundation of CUNY.

(C) 2004 Kluwer Academic Publishers. Printed in the Netherlands. 
relation. Let $\mathbf{L}$ be a (finitary) language and let $\sim$ be a relation between sets of well-formed formulas (wffs) $\Delta$ and single wffs $a(\Delta \sim a)$ which means that $a$ is a non-monotonic consequence of $\Delta$. In (Gabbay, 1985) Gabbay proposed three minimal constraints that a supra-classical ${ }^{1}$ consequence relation, whether monotonic or non-monotonic, should obey.

$$
\begin{aligned}
& a \sim a \quad(\mathrm{CUT}) \frac{\Delta, a \sim b, \Delta \sim a}{\Delta \sim b} \\
& (\mathrm{CM}) \frac{\Delta \sim a, \Delta \sim b}{\Delta, a \sim b}
\end{aligned}
$$

Reflexivity and CUT are self-explanatory. Cautious monotony (CM) is a restricted form of monotony. A supra-classical consequence relation on $\mathbf{L}$ obeying R, CUT and CM is usually called cumulative and is denoted $\mathbf{C}$. Gabbay defended the adequacy of $\mathbf{C}$ mainly in terms of proof-theoretical considerations.

Different semantics have since then been proposed for C. David Makinson offered a first model in (Makinson, 1989). Then Kraus et al offered in (Kraus, Lehmann and Magidor, 1990) a comprehensive semantical analysis of several extensions of $\mathbf{C}$ by appealing to the preferential semantics of Shoham (Shoham, 1987). Finally Lehmann and Magidor studied in (Lehmann and Magidor, 1990) one of the strongest preferential systems, the rational system $\mathbf{R}$.

The semantical approach used in preferential models is reminiscent of the semantical techniques used in some branches of conditional logic. In particular preferential models and possible worlds models of conditionals share some structure (see (Cross and Nute, 1998)). Nevertheless the relations between these two approaches are more intricate than might seem at first sight. An obvious difference is that conditional logic enriches the object language by adding a binary connective $>$ and giving axioms for it, while non-monotonic logic operates by adding a new consequence relation to the standard one and postulating properties for it. Several interesting correspondences between the two approaches have nevertheless been established in recent papers (see, for example, (Arló-Costa and Shapiro, 1992), (Delgrande, 1987), (Katsuno and Satoh, 1996)). Most of this work focuses on finding useful

\footnotetext{
${ }^{1}$ Supra-classical means that $\vdash \subseteq \mathcal{C}$, where $\vdash$ is the classical notion of consequence.
} 
model-theoretical correspondences and studying the appropriate nonnested conditionals. This approach is, nevertheless, only one of several different (and independently influential) manners of studying defeasible reasoning.

A rival approach treats non-monotonic relations as epistemic conditionals (see the corresponding section in (Cross and Nute, 1998)) characterizable via some variant of the so-called Ramsey test:

(Ramsey) $a \sim_{E} b$ if and only if $b \in \rho(E) * a$

where $a \sim_{E} b$ indicates that $a \sim b$ holds with respect to the epistemic state $E, \rho(\mathrm{E})$ are the expectations associated with $E$, and $\rho(E) * a$ is the result of supposing that $a$ is the case with respect to the state $E$. Intuitively, according to this approach, non-monotonic relations are about conditional expectations:

(P) $a \sim_{E} b$ if and only if as many as possible of the expectations associated with $E$ as are compatible with $a$, together with $a$, entail $b$.

The underlying idea is that accepting $a \sim b$ in $E$ is tantamount to accepting that $b$ is expected upon supposing that $a$ (i.e., from the point of view of $\rho(E) * a)$. This idea has been technically articulated in recent papers. Some models are purely qualitative in nature (see (Gärdenfors and Makinson, 1994)), while others offer a decision-theoretical account of the nature of the expectations used in (P) (Levi, 1996). Different types of Ramsey clauses are obtained by focusing on different possible representations of the epistemic state E (as a logical theory, an ordinal conditional function, etc). It should be remarked, nevertheless, that the models offered in (Gärdenfors and Makinson, 1994) and (Gärdenfors, 1993) do not appeal to a decision-theoretically motivated notion of expectation. Peter Gärdenfors is quite clear about this in (Gärdenfors, 1993):

The word 'expectation' as it is used in this paper should thus not be confused with the notion of 'expected utility' in decision theory. 'Expected utility' has to do with expectations of the values of various outcomes, while the notion of expectation studied here concerns beliefs about the world. In my opinion, this use of 'expectation' comes much closer to the everyday use ((Gärdenfors, 1993), p. 5).

Finally non-monotonic consequence relations have also been characterized in terms of probabilistic models. This semantic framework treats conditionals as syntactic carriers of probability, rather than as carriers of truth (Adams, 1975). The basic idea in this tradition is that the 
probability of a simple, or un-nested conditional $a>b$ (corresponding in the language to $a \sim b$ ) is given by the probability of (the proposition corresponding to) its consequent, $b$, conditional in its antecedent, $a .^{2}$ The most sophisticated presentation of this idea appeals to a generalized notion of conditional probability where it is also possible to condition on events of zero measure. Let $A, B, \ldots$ denote the propositions expressed by $a, b, \ldots$.

(Ramsey-Adams-McGee) $a \sim_{P} b$ if and only if $\mathrm{P}(B \mid A)=1$

This modeling of defeasible consequence is 'prima facie' unconnected with expectation tests. A bridge between conditional probability and the qualitative notions of expectation and full belief is needed in order to articulate this connection. It is well-known, nevertheless, from (Kyburg, 1961), that it is hard to construct sound bridges linking probability and qualitative belief. ${ }^{3}$ Some obvious solutions, like defining full belief as unconditional measure one are known to be deeply defective. In this paper we appeal to a slight modification of a proposal presented by Bas van Fraassen in (van Fraassen, 1995). This approach permits us to construct a reasonable definition of both expectations and full beliefs for a generalized conditional probability function. With the help of these definitions we can use the following instance of $(\mathrm{P})$ :

(P) $a \sim_{P} b$ if and only if $A$, together with as many as possible of the qualitative expectations associated with $P$ which are compatible with $A$, classically entails $B$.

The probabilistic model of expectations presupposed by $(\mathrm{P})$ helps to provide foundations for the primitive notion of expectation used in (Gärdenfors and Makinson, 1994) and (Gärdenfors, 1993). Even when the notion does not have an obvious decision-theoretical origin,${ }^{4}$ it is possible to articulate it in terms of the notion of primitive conditional probability used in the proposed extension of the RamseyAdams acceptability tests for conditionals. This links two areas of

\footnotetext{
2 The parenthetical remark concerns the issue of what are the carriers of probability. Parenthetical additions are needed if the carriers of probability are propositions. Here we will adopt this view. Some of the standard probabilistic models, nevertheless, prefer to use sentences as probability carriers. This is perhaps motivated by the goal of developing not only probabilistic models of modalities and conditionals, but also probabilistic models of classical logic as well, an issue with which we will not be concerned here.

3 As is well-known that it is hard to construct bridges linking probability of conditionals and conditional probability (Lewis, 1976).

4 Arguments for the impossibility of providing decision-theoretic reconstructions of some forms of defeasible inference are presented in (Levi, 1996).
} 
research on models of non-monotonic consequence, which, up to now, have remained isolated from each other.

The probabilistic model offered here differs in important ways from previous probabilistic accounts of rational inference, like the one produced by Judea Pearl in (Pearl, 1988) and by Lehmann and Magidor in (Lehmann and Magidor, 1990). In fact, the first appeals to extremely high probability and the second to non-standard probability. Our model uses a simpler account in terms of primitive conditional probability. On the other hand, unlike other models in terms of conditional probability (Hawthorne, 1998), we link our model with previous qualitative accounts in terms of expectations. The construction of this link depends on our choice of primitives in building the probabilistic models. We should mention as well that our completeness results for the system $\mathcal{R}$ of Lehmann-Magidor are valid both for finitary and infinitary languages. The consideration of richer languages allows us to investigate the role of countable additivity in probabilistic models of conditionals. We show that a rational logic defined over a logically infinite language can only be fully characterized in terms of finitely additive conditional probability.

\section{Technical results}

Our model uses two-place probability functions, i.e. we adopt basic axioms for conditional probability, rather than defining it in the usual way from monadic probability. ${ }^{5}$ Bas van Fraassen developed in (van

\footnotetext{
5 The terminology 'Popper functions' is sometimes used to refer to these two place functions. This terminology is somewhat misleading. Popper was only one among many researchers who have proposed to take conditional probability as an irreducible primitive. And these accounts do not exhaust all approaches capable of representing conditioning with zero measure events. The traditional Kolmogorovian account is already capable of doing this.

Karl Popper offered an account of primitive conditional probability in (Popper, 1968). His main intention was to characterize the notion of logical consequence in probabilistic terms. Popper employed axioms previously used by Harold Jeffreys in (Jeffreys, 1961). But Popper's proposal was preceded by the work of Bruno De Finetti (De Finetti, 1936) who also defended a variant of the view that all probability is in fact conditional probability. Nevertheless, for reasons that we cannot discuss at length here, De Finetti's account depends on assuming as basic a qualitative notion of certainty. If one uses terminology that has some philosophical currency, De Finetti was not a radical probabilist. I.e. he appealed to a non-probabilistic primitive (certainty) in his characterization of conditional probability. Here we will adopt the viewpoint of radical probabilism, given that this is the approach taken by contemporary defenders of probabilistic semantics. Finally Alfred Renyi (Renyi, 1970) was also a proponent of the view that all probability is really conditional in nature. His work had a more direct connection with the mainstream of contemporary
} 
Fraassen, 1995) a probabilistic definition of full belief in terms of such functions.

We offer a modified and extended version of van Fraassen's model of (van Fraassen, 1995) and use it to model Bayesian update. (van Fraassen, 1995) studies two place probability functions $P(. \mid$.) defined on a $\sigma$-field $F$ over some set $U$. The requirements are that

(I) for any fixed $A$, the function $P(X \mid A)$ as a function of $X$ is either a (countably additive) probability measure, or has constant value 1 .

(II) $P(B \cap C \mid A)=P(B \mid A) P(C \mid B \cap A)$ for all $A, B, C$ in $F$.

If $C \subseteq B \subseteq A$, then (II) above can be simplified as

(II') $P(C \mid A)=P(C \mid B) P(B \mid A)$.

The probability (simpliciter) of $A, \operatorname{pr}(A)$, is $P(A \mid U)$. We will follow established terminology by referring to (II) as the Multiplication Axiom. ${ }^{6}$

For fixed $A$, if $P(X \mid A)$ is a probability measure as a function of $X$, then $A$ is normal and otherwise it is abnormal - $P(X \mid A)$ has constant value 1 , and, in particular, $P(\emptyset \mid A)=1$. Now in fact a normal set may have measure 0 in which case we do not expect to believe it, but we still might. E.g. the rationals as a subset of the reals have measure 0, but if we pick a real at random and it turns out to be rational we do not throw up our hands in despair! Thus the rationals $Q$ are a normal set of measure $0, P(\emptyset \mid Q)=0$. An abnormal set by contrast not only has measure 0 , but it is so small that we do not know what to believe if we believe in that. Indeed, if $A$ is abnormal, then $P(\emptyset \mid A)=1$. Thus $A$ is normal iff $P(\emptyset \mid A)=0$. van Fraassen shows in (van Fraassen, 1995) that supersets of normal sets are normal and that subsets of abnormal sets are abnormal. Assuming that the whole space is normal, abnormal sets must have measure 0 . For if $X \subset Y, Y$ is normal and $X$ is abnormal, then we have $P(\emptyset \mid Y)=P(\emptyset \mid X) P(X \mid Y)$. Since $P(\emptyset \mid Y)=0$ and $P(\emptyset \mid X)=1$, it must be the case that $P(X \mid Y)=0$. However, of course the converse need not hold, a normal set may also have measure 0 . We now show the following:

theory of probability. In this paper we use the axioms proposed in (van Fraassen, 1995), but we will also study models where monadic probability is finitely additive. Those models are not considered in (van Fraassen, 1995). Therefore we prefer to use the more neutral terminology of 'two-place probability functions'.

6 This axiom appears under the name 'W. E. Johnson's product rule' in (Jeffreys, 1961). 
LEMMA 2.1. A countable union of abnormal sets is abnormal, though an intersection of normal sets, even a finite intersection, need not be normal.

PROOF 2.1. The second part is easy. Let $X$ and $Y$ be disjoint normal sets (e.g. disjoint sets with positive measure) and $W$ an abnormal set. Then since $X \cup W$ and $Y \cup W$ contain the normal sets $X, Y$ respectively, they are both normal. But their intersection is $W$ which is by assumption not normal.

Coming to the first part, let us show first that the the union of two abnormal sets $X, Y$ is still abnormal. Note that $P(\emptyset \mid X \cup Y)=$ $P(\emptyset \mid X) P(X \mid X \cup Y)$ from the multiplication axiom. Since $X$ is abnormal, $P(\emptyset \mid X)=1$. Thus if $X \cup Y$ were normal, then $P(\emptyset \mid X \cup Y)=0$ and since $P(\emptyset \mid X)=1, P(X \mid X \cup Y)$ must be 0 .

By a symmetric argument, $P(Y \mid X \cup Y)=0$. This is a contradiction since $X, Y$ make up all of $X \cup Y$.

To come to the countable union case, suppose that $X_{i}: i \in N$ are countably many abnormal sets and by the argument above, we can suppose that $i<j \rightarrow X_{i} \subset X_{j}$. Let $Z=\bigcup X_{i}$. Now we know that if $Z$ were normal, then for all $i, P\left(X_{i} \mid Z\right)=P(\emptyset \mid Z)=0$. But then continuity (a consequence of countable additivity) yields $P(Z \mid Z)=0$ which is absurd whether $Z$ is normal or abnormal.

The last part of the previous proof depended on Countable Additivity, while the first part did not. In the following we shall confine ourselves to the case where the whole space $U$ is normal. The notion of normality is closely connected to an epistemic analysis of the notion of a priori: (A) $A$ is a priori for $P$ iff $P(A \mid X)=1$ for all $X$, iff $U-A$ is abnormal for $P$. For if $U-A$ were normal for $P$ then we would have $P(A \mid U-A)=P(A \cap(U-A) \mid U-A)=P(\emptyset \mid U-A)=0$.

Slightly modifying van Fraassen's definition we define a core as a set $K$ which is normal and satisfies the strong superiority condition (SSC) i.e. if $A$ is a nonempty subset of $K$ and $B$ is disjoint from $K$, then $P(B \mid A \cup B)=0$ (and so $P(A \mid A \cup B)=1$ ). ${ }^{7}$ Thus any non-empty subset of $K$ is more "believable" than any set disjoint from $K$. It can then be established that all non-empty subsets of a core are normal.

LEMMA 2.2. (Finesse): all non-empty subsets of $K$ are normal.

PROOF 2.2.

Suppose that $K$ is a core and $A$ a nonempty subset of it. Let $B=\emptyset$. Then $B$ is disjoint from $K$ and hence, by $S S C, P(B \mid A)=P(B \mid A \cup B)=$ 0 . Thus $A$ is normal.

\footnotetext{
${ }^{7} \mathrm{~A}$ is superior to $\mathrm{B}$ if $\mathrm{P}(\mathrm{A} \mid \mathrm{A}+\mathrm{B})=1$, where + is symmetric difference.
} 
van Fraassen's characterization of cores requires a slightly different superiority condition, and the property called (A3) according to which the complement of a core should be normal. There might be arguments in favor of adopting this point of view. For example, if the union of cores represents the strongest proposition fully believed by the agent, one might want to preserve the possibility of genuine revisions of this body of belief with normal propositions. ${ }^{8}$ Nevertheless, the condition introduces certain asymmetries in the resulting probabilistic models that we prefer to avoid. van Fraassen's solution entails that if we revise with the largest core, such a core cannot be among the cores of the updated function. Since we are interested in updating, we prefer to avoid this possibility. Further arguments in favor of our reformulation will be offered later.

Formally many of the basic theorems proved by van Fraassen and other scholars who worked in this area can be proved in our modified framework with minor changes. The most salient results will be listed below.

LEMMA 2.3. The family of cores induced by a two place probability function $P$ is nested, i.e. given any two cores $K_{1}, K_{2}$ either $K_{1} \subseteq K_{2}$ or $K_{2} \subseteq K_{1}$ (vanFraassen, 1995).

Indeed we have the following important fact:

THEOREM 2.1. (Descending Chains). The chain of belief cores induced by a non-coreless 2-place function P cannot contain an infinitely descending chain of cores.

A proof of this fact was already given in (Arló-Costa, 1999), but since it is short we repeat it here for the sake of completeness. Suppose that $K_{i}: i \in N$ is a strictly descending sequence of cores. Let $p_{i} \in K_{i}-K_{i+1}$ and let $X_{i}=\left\{p_{i}, p_{i+1}, \ldots\right\}$. Each $p_{i}$ then dominates $p_{j}$ for $j<i$ since $p_{i}$ is in the core $K_{i}$ and $p_{j}$ is not. Thus we get $P\left(X_{i} \mid X_{1}\right)=1$ for all $i$. But then by continuity, $P\left(\cap X_{i} \mid X_{1}\right)=1$ which is absurd as $\bigcap X_{i}$ is empty and $X_{1}$ is normal.

Cores are well ordered under inclusion and closely resemble Grove spheres (Grove, 1988) and Spohn's ordinal conditional functions (Spohn, 1988). Indeed we can show that for any successor ordinal $\alpha$ there is a space $(U, F, P)$ such that the family of its cores has ordinal $\alpha$. Let $\alpha$ be any (von Neumann) ordinal. Then $\alpha=\{\beta \mid \beta<\alpha\}$. For subsets $X, Y$

8 This might be so even when van Fraassen's account does not give us the tools to perform such a revision. Also for him the union of cores represents a proposition accepted by the agent rather than its full beliefs. 
of $\alpha$ with $X \subseteq Y$ let $P(X \mid Y)$ equal 1 if $\min (X)=\min (Y)$ and equal 0 otherwise, i.e. if $\min (Y)<\min (X)$. Then all non-zero ordinals $<\alpha$ are clearly cores, and for the sake of symmetry, as we indicated above, we include $\alpha$ also as a core.

LEMMA 2.4. There is a smallest as well as a largest core. Moreover, the smallest core (and hence every core) has measure 1.

PROOF 2.3. Since cores are well ordered by inclusion, there is naturally a smallest core. There is also a largest core, namely the union of all cores. To see that this union, say $K$, is also a core, note first that if $X$ is a nonempty subset of $K$ then there must exist a core $K^{\prime}$ which overlaps $X$. Now the subset $X \cap K^{\prime}$ of $K^{\prime}$ is normal and thus $X$ is also normal. To see superiority we use a similar argument. Let $X, K^{\prime}$ be as before and let $B$ be disjoint from $K$. Then $B$ is also disjoint from $K^{\prime}$ and hence $X \cap K^{\prime}$ is superior to $B$. Hence so is $X$ itself. That even the smallest core $K_{0}$ has measure one follows from the fact that $K_{0}$ is superior to its complement and together they make up measure 1. Thus all cores have measure 1.

We propose that the smallest core be identified with (ordinary) beliefs or expectations and the largest core with full beliefs, (i.e. a priori beliefs) so that in general probability 1 is not sufficient for full belief. Arguments supporting this interpretation are offered in the following section. The resulting notion of full belief is quite strong epistemologically - but rather weak logically. In fact, robustness under arbitrary suppositions (encoded as aprioricity for $\mathrm{P}$ ) is both a necessary and a sufficient condition for full belief for P. Unlike most of the recent literature on belief change, which focuses on (full) belief revision, the probabilistic framework we are studying is unable to characterize such revisions. ${ }^{9}$ It really studies inductive expansions of the given body of full beliefs. Or, under a different point of view, revisions of (ordinary) beliefs or expectations .

\section{The countable case}

If the set $U$ is countable then very nice properties hold. There is a set of heavy points $p$, i.e., those $p$ such that $P(\{p\} \mid U)>0$, all of which are normal. There may also be normal (light) points $p$ such that $\operatorname{pr}(\{p\})=$ 0 . These will be normal because $P(\emptyset \mid\{p\})=0$. Finally there may be abnormal points $p$ such that $\operatorname{pr}(\{p\})=0$ and $P(\emptyset \mid\{p\})=1$.

\footnotetext{
${ }^{9}$ van Fraassen's unmodified theory makes these revisions entertainable, but it gives no guidance for implementing them.
} 
LEMMA 3.1. (Heavy Points) Let $S=\langle U, F\rangle$ be a countable space and $P(. \mid$.$) a two-place probability function defined on the space. The set$ $H(P)$ of heavy points of $P$ constitute the smallest core, which, moreover, has measure 1.

\section{PROOF 3.1.}

We already showed that the smallest core has measure 1. Note that no heavy point can be dominated, hence the smallest core contains at least the heavy points. Moreover, any set of heavy points is normal, and finally any non-empty set of heavy points is superior to any set of light points, whether normal or abnormal.

Remark: the proof we just gave makes use of the countability of $U$, and will not carry over to the uncountable case where various intuitions begin to clash.

Let the ordering $<$ on $U$ be defined for all (distinct) pairs of points $\mathrm{p}$, $\mathrm{q}$, such that $\{\mathrm{p}, \mathrm{q}\}$ is normal, by $p<q \leftrightarrow P(\{p\} \mid\{p, q\})=1$, i.e. if we know that we have picked one of $p, q$ then it must be $p$. Similarly, let $p \cong q \leftrightarrow 0<P(\{p\} \mid\{p, q\})<1$. When $\{\mathrm{p}, \mathrm{q}\}$ is abnormal, we set $p \cong q$ always, and all normal points dominate abnormal points. Then $<$ is a well quasi-ordering with $\cong$ as its associated equivalence relation and the cores are just sets of normal points closed under $\leq$. The heavy points are all equivalent to each other and in the smallest core. The light normal points are superior to the abnormal points but may have superiority relations among themselves, as the ordinal example above shows. In that example 0 was the only heavy point, but all other points, though light, were normal.

For a more general example, let $\alpha$ be any countable ordinal strictly greater than $\omega$. We let $P(X \mid Y)=1$ if $\min (\mathrm{X})=\min (\mathrm{Y})<\omega \cdot P(X \mid Y)$ $=0$ if $\min (\mathrm{Y})<\omega$ and $\min (\mathrm{Y})<\min (\mathrm{X})$. And finally $P(X \mid Y)=1$ if $\min (\mathrm{Y}) \geq \omega$. Now 0 will be the only heavy point, all finite points will be light but normal, and all points greater than or equal to $\omega$ will be abnormal. A set will be normal iff it contains a finite ordinal.

The fact that (in the countable case) the heavy points constitute the smallest core (which has measure 1), gives support to one of our proposed modifications of van Fraassen's account. In fact, one of the main purposes of the use of two-place functions in the definition of full belief is to avoid a problematic identification of belief and probability one (which yields the so-called (infinite) lottery paradoxes - see (Kyburg, 1961)). But such a problematic identification would be required if we interpreted the innermost core of $\mathrm{P}$ as an encoding of the body of P's full beliefs (in the countable case). By the same token, we did not want to make an identification of null measure with epistemic impossibility. It seems more natural to say that P's full beliefs are given by the 
union of P's cores, rather than by their intersection. The intersection of cores can, in turn, be seen as the representation of P's plain beliefs (or qualitative expectations). The following example tries to provide more intuitive clues supporting this idea. The example illustrates as well the interest of adopting our modified characterization of cores.

Example: The sample space has a countable set of atoms, resulting from the following: in independent trials, a fair coin is flipped until we get a head, and then the trials stop. The set of possible outcomes is indexed by the number of tails $X=(0,1,2, \ldots ., n, \ldots, \omega)$, where $\omega$ designates never stopping, i.e., flipping forever and seeing only tails. Evidently $\operatorname{pr}(X$ is finite $)=1$ and $\operatorname{pr}(X=n)=\left(\frac{1}{2}\right)^{(n+1)},(n=0,1, \ldots)$, so that (obviously) $\operatorname{pr}(X=\omega)=0$, and this is the only null event, apart from the impossible event. It seems unreasonable in this case to require that a rational agent modeled by $P$ ought to fully believe that $X$ is finite.

Notice that in this case it is not enough to interpret full beliefs as the union of cores, as long as cores are defined in terms of an unmodified version of van Fraassen's original definition. In fact, according to that definition, $P$ induces a unique core $U-\{\omega\}$. Van Fraassen's condition (A3) impedes considering the entire space as a core. Our modified definition makes this limit case more intuitive. According to our definition, $P$ induces two cores, $U-\{\omega\}$, and $U$. It is also reasonable to say, in the case under consideration, that a finite outcome is expected, rather than fully believed. This intuition is captured by our characterization of qualitative expectations as the intersection of cores.

Returning to our previous example of an ordinal greater than $\omega$, note that if we were to allow ordinals greater than $\omega$ to be possible answers, then a finite ordinal would represent an event of positive probability, $\omega$ would be a normal event of 0 probability, but an ordinal strictly greater than $\omega$ would be an abnormal event for we would not know what to make of the report that the coin landed heads $\omega+1$ times. $^{10}$

If we learn some proposition $A$, even one whose probability $\operatorname{pr}(A)=$ 0 , then as long as $A$ intersects the largest core $\mathcal{N}, P$ relativised to $A$ has again an elegant theory and its cores are essentially the old cores intersected with $A$. Thus "revising" by $A$ is well defined. A detailed axiomatic presentation of the notion of belief change that thus arises is presented both in (Arló-Costa, 2001) and (Arló-Costa and Thomason, 2001). We do not need to go through these details here, aside from

\footnotetext{
10 Even the statement that the coin landed heads $\omega$ times requires some interpretation. But we could certainly make sense of someone's belief that the coin will land heads $\omega$ times, i.e. always.
} 
noticing that the innermost core of $P(X \mid Y \cap A)$ encodes as many as possible of the expectations of $\mathrm{P}$ as are compatible with $\mathrm{A}$.

\section{Countable Core Logic and Rational Logic}

In (Kraus, Lehmann and Magidor, 1990), Kraus, et al, present the following definition of a preferential model:

DEFINITION 4.1. If $P \subseteq S$ and $<i$ is a binary relation on $S, P$ is a smooth subset of $S$ iff $\forall t \in P$, either there exists an $s$ minimal in $P$ such that $s<t$ or $t$ is itself minimal in $P$.

DEFINITION 4.2. A preferential model $W$ for a universe $U$ is a triple $\langle S, l,<\rangle$ where $S$ is a set, the elements of which will be called states, $l: S \rightarrow U$ is a labelling function which assigns a world from the universe of reference $U$ to each state and $<$ is a strict partial order on $S$ (i.e., an irreflexive, transitive relation) satisfying the following smoothness condition: for all a belonging to the underlying propositional language $L$, the set of states: $\hat{a}=\{s: s \in S, s \equiv a\}$ is smooth; where $s \equiv$ a (read $s$ satisfies a) iff $l(s) \models a$, where ' $=$ ' is the classical notion of logical consequence.

The definitions introduced above allow for a modification of the classical notions of entailment and truth compatible with the idea of nonmonotonicity. The following definition shows how this task can be done:

DEFINITION 4.3. Suppose a model $W=\langle S, l,<\rangle$ and $a, b \in L$ are given. The entailment relation defined by $W$ will be denoted by $\sim_{W}$ and is defined by: $a \sim_{W} b$ iff for all $s$ minimal in $\hat{a}, s \equiv b$.

Preferential models were used in (Kraus, Lehmann and Magidor, 1990) to define a family of preferential logics. In (Lehmann and Magidor, 1990), Lehmann and Magidor focused on a subfamily of preferential models - the so-called ranked models.

DEFINITION 4.4. A ranked model $R$ is a preferential model $\langle S, l,<\rangle$ where the strict partial order $<$ is defined in the following way: there is a totally ordered set $W$ (the strict order on $W$ will be denoted by $\angle$ ) and a function $r: S \rightarrow W$ such that $s<t$ iff $r(s) \angle r(t)$.

The effect of the function $r$ is to rank the states, i.e. a state of smaller rank is more normal than a state of higher rank. The intuitive 
idea being that for $r(s)=r(t)$ the sates $s$ and $t$ are at the same level in the underlying ordering. In order to increase intuition about ranking it is useful to notice that, if $<$ is a partial order on the set $T$ the ranking condition presented above is equivalent to the following property:

(Negative Transitivity) For any $s, t, u$ in $T$, such that $s<t$, either $u<t$ or $s<u$.

Lehmann et al also introduce ranked models where the ordering of the states does not need to obey the smoothness requirement.

DEFINITION 4.5. A rough ranked model $V$ is a preferential model $\langle S, l,<\rangle$ for which the strict partial order $<$ is ranked and the smoothness requirement is dropped.

From the syntactical point of view, Kraus et al. proved a representation theorem for the following system $\mathcal{P}$ in terms of the above preferential models.

$$
\begin{aligned}
& \text { (R) } \quad a \sim a \\
& (\mathrm{LLE}) \frac{\models a \leftrightarrow b, a \sim c}{b \sim c} \\
& (\mathrm{RW}) \frac{\models a \rightarrow b, c \sim a}{c \sim b} \quad(\mathrm{CM}) \frac{a \sim b, a \sim c}{a \wedge b \sim c} \\
& (\mathrm{AND}) \frac{a \sim b, a \sim c}{a \sim b \wedge c} \quad(\mathrm{OR}) \frac{a \sim c, b \sim c}{a \vee b \sim c}
\end{aligned}
$$

LLE stands for 'left logical equivalence', RW for 'right weakening' and CM for 'cautious monotony'. Lehmann, et al., prove that the system $\mathcal{R}$, complete with respect to ranked models, can be obtained by adding the following rule of rational monotony to the above set of rules.

$$
(\mathrm{RM}) \frac{a \sim c, \neg(a \sim \neg b)}{a \wedge b \sim c}
$$

Naturally, if (RM) is added then (CM) is no longer necessary. Lehmann et al. suggested in (Lehmann and Magidor, 1990) that the syntactic system $\mathcal{R} \mathcal{R}$ obtained from $\mathcal{R}$ by dropping the rule (CM) is sound 
and complete with respect to rough ranked models. They obtain this conjecture from the work of James Delgrande in conditional logic. This conjecture was proved true in (Arló-Costa and Shapiro, 1992).

\subsection{Countable Core Logic and Probabilistic Models}

$\mathrm{S}=\langle U, F\rangle$ is a probabilistic space, with $\mathrm{U}$ countable and where $\mathrm{F}$ is a Boolean sub-algebra of the power set of $U$. The assumption about the size of $U$ cannot be dispensed with. It will be maintained throughout this paper.

DEFINITION 4.6. $M=\langle S, P, V\rangle$ is a probabilistic model, if $S=$ $\langle U, F\rangle$ is a probabilistic space, $U$ is a countable set, and $F$ is a Boolean sub-algebra of the power set of $U . V$ is a classical valuation mapping sentences in $L$ to measurable events on $F$ and $P$ is a two-place function on $U$ obeying:

(I) for any fixed $A$, the function $P(X \mid A)$ as a function of $X$ is either a (finitely additive) probability measure, or has constant value 1.

(II) $P(B \cap C \mid A)=P(B \mid A) P(C \mid B \cap A)$ for all $A, B, C$ in $F$.

As we did above, we will abbreviate $V(a)$, for $a \in \mathrm{L}$, as $A$. Of course, $V$ is defined inductively as usual for arbitrary formulae in L. So we will use the aforementioned abbreviation only when there is no possible ambiguity.

DEFINITION 4.7. A probabilistic model is countably additive ( $C A$ ) iff for any fixed $A$, the function $P(X \mid A)$ as a function of $X$ is either a countably additive probability measure, or has constant value $1 .{ }^{11}$

Let the ordering $<$ on $U$ be defined by $p<q$ if and only if $P(\{p\} \mid\{p, q\})=$ 1 , i.e. if we know that we have picked one of $p, q$ then it must be $p$. Similarly, let $p \cong q$ if and only if $0<P(\{p\} \mid\{p, q\})<1$. From now on we will call the ordering $<$ induced by a probabilistic model $\mathrm{M}$ the ranking ordering for M. notice that as a corollary of Theorem 2.1 stated above:

LEMMA 4.1. The ranking ordering $<$ for a $C A$ probabilistic model $M$ is well-founded.

Now we can define: $a \sim_{<M} b$ iff for every $u \in \mathrm{U}$, such that $u$ is minimal in $A$, according to the ranking ordering for $\mathrm{M}, u \in B$. Notice that the set of minimal A-points, according to the ordering induced by $P$ in

\footnotetext{
11 Notice that CA is stronger than the simpler assumption that $P(X \mid U)$ is either a countably additive measure or has constant value 1 .
} 
$M$, is the innermost core of $P(X \mid Y \cap A)$, encoding as many as possible of the expectations of $P$ as are compatible with $A$. So, $a \sim_{<M} b$ iff as many as possible of the expectations of $P$ as are compatible with A, entail B. So, here we have an expectation test derived probabilistically from conditional probability.

It is important to notice that there is an alternative probabilistic definition of $\sim$. Such a definition requires that: $a \sim_{P M} b$ iff $P(B \mid A)=$ 1. These two ways of defining a supraclassical consequence relation are intimately related, but we will verify below that they do not coincide in all cases. We have nevertheless that:

LEMMA 4.2. For a CA probabilistic model $M$, $a \sim_{P M} b$ iff $a \sim_{<M} b$

PROOF 4.1. In order to prove this result it is enough to show that if the universe $U$ of the underlying space is countable and normal for a function $P$, then for all propositions $A, B, P(B \mid A)=1$ if and only if the smallest core of $P[A](X \mid Y)=P(X \mid Y \cap A)$ entails $B$. The lemma of Descending Chains and the lemma of Heavy Points are enough to prove this result, which appears in (Arló-Costa, 2001). We reproduce it here for the sake of completeness. Let $\operatorname{Ex}(P)$ denote the expectations of $P$, i.e. the innermost core of $P$; and let $F(P)$ denote the full beliefs of $P$, i.e. the outermost core of $P$. Assume $E x(P[A]) \subseteq B$. Then if $F(P) \cap A$ $\neq \emptyset$ we have that $\operatorname{Ex}(P[A])$ is non-empty (by the lemma of descending chains) and $P[A](E x(P[A]) \mid U)=1$. Therefore, the assumption yields $P(\operatorname{Ex}(P[A]) \mid A)=1=P(B \mid A)$. When $F(P) \cap A=\emptyset, P[A]$ is abnormal. Therefore, $P(X \mid A)=1$ for all $X$. In particular $P(B \mid A)=1$. Assume now $P(B \mid A)=1$. Then $\operatorname{Ex}(P)$ is the support $S(P)=\{x \in U: P(x)>0\}$ - by the lemma of heavy points. So, if $A \cap F(P) \neq \emptyset$ we have $\operatorname{Ex}(P[A])$ $=S(P[A])$. Assume then by contradiction that Ex $(P[A])$ is not included in $B$. The proof can then be completed by cases. First, consider the case $\operatorname{Ex}(P[A]) \cap B$ is empty. In this case, for all $y$ in $B, P[A](y \mid U)=0-$ again by heavy points. Now, since the space is countable and normal for $P$, countable additivity guarantees that 0 equals the sum over $y$ of all values $P[A](y \mid U)=P[A](B \mid U)=P(B \mid A)=1$. Contradiction. Second, consider the case when $\operatorname{Ex}(P[A]) \cap B$ is non-empty. In this case there is a strict subset $Z$ of $\operatorname{Ex}(P[A])$, such that $1=P(B \mid A)$ $=P(Z \mid A)<1$. Contradiction. Third, in order to finish the proof, we should consider the case $A \cap F(P)$ is empty. This case is immediate, because by definition $\operatorname{Ex}(P[A])=\emptyset \subseteq B$.

Some results about rational logic follow immediately from previous results. In particular we have a soundness result for $\mathcal{R}$ in terms of CA models: 
LEMMA 4.3. For a CA probabilistic model $M$, if $a \sim_{P M} b$ (or $a \sim_{<M} b$ ), then $a \sim_{R} b$.

From now on it will be important to make precise distinctions about the nature of the underlying language $L$ used to define non-monotonic relations. If the set of primitive propositional variables used in the definition of $L$ is finite, we will call the language logically finite. Now, with the proviso that $L$ is logically finite we have a completeness result for Rational Logic in terms of CA Probabilistic Models. Some preliminary definitions and intermediate lemmas are needed in order to state the result.

DEFINITION 4.8. Let $M=\langle S, l,<\rangle$ be a ranked model. Its associated spheres model is a triple $\langle S, l, \mathbf{S}\rangle$ where $S$ is $M$ 's universe of states, $l$ is $M$ 's original labeling function, and $\mathbf{S}=\left\{S_{x}: x \in S\right\} \cup S$, where $S_{x}$ $=\{y \in S: y<x\}$

Since ranked models are smooth, we know that for every $a \in \mathrm{L}$, there is a minimal $\hat{a}$-sphere of states. Let the intersection of $\hat{a}$ with this sphere be called $S_{m, a}$. Therefore we can define that $a \sim_{R} b$ is satisfied in the associated spheres model corresponding to $\mathrm{M}=\langle S, l,<\rangle$ as long as for every $s$ in $S_{m, a}$ we have that $s \equiv b$.

It is important to notice that ranked models and their associated spheres models can be correlated one-to-one. Therefore we can establish the following useful result regarding the validity of Generalized Horn Patterns (GHP) of the following form: If $a_{1} \sim b_{1}, \ldots, a_{i} \sim b_{i}, \ldots, a_{n} \sim b_{n}$, then $a \sim b .^{12}$

LEMMA 4.4. A GHP is valid in a ranked model $M=\langle S, l,<\rangle$ if and only if it is valid in its associated spheres model $M^{\prime}=\langle S, l, \mathbf{S}\rangle$

PROOF 4.2. Essentially we need to show that every conditional assertion of the form $a \sim b$, is validated in a ranked model $M$ if and only if it is validated in its associated spheres model $M$ '. $a \sim b$ is valid in $M$ $\langle S, l,<\rangle$ if and only if for every state $s$ in $M_{<, a}=\{s \in S: s \in \hat{a}$ and for all $\left.s^{\prime} \in \hat{a}, \neg\left(s^{\prime}<s\right)\right\}$, we have that $s \equiv b$.

On the other hand $a \sim b$ is valid in the associated spheres model $M$ ' $=\langle S, l, \mathbf{S}\rangle$ if and only if for every state $s$ in $S_{m, a}=\hat{a} \cap(\cap\{S \in \mathbf{S}$ : $S \cap \hat{a} \neq \emptyset\})$. we have that $s \equiv b$.

So, in order to complete the proof we have to show that $S_{m, a}=M_{<, a}$. Assume that $s \notin S_{m, a}$, but $s \in M_{<, a}$. Therefore there exists a sphere $T \in \mathbf{S}$, such that $T \cap \hat{a} \neq \emptyset$ and $s \notin T \cap \hat{a}$.

\footnotetext{
12 The techniques used here in order to eliminate states from ranked models follow a slightly different (an improved) presentation of ideas first published in (Arló-Costa and Shapiro, 1992).
} 
We need to pause here for a moment. Notice that for every ranked model $M=\langle S, l,<\rangle$ and its associated spheres model $M^{\prime}=\langle S, l, \mathbf{S}\rangle$, there is a simple manner of defining a relation $<^{\prime}$ from $M^{\prime}$, in such a way that $<=<$.

$(S$ to $<) s<^{\prime} t$ if and only if there is $V \in \mathbf{S}$ and $s \in V$ and $t \notin V .{ }^{13}$

Consider first $<\subseteq<^{\prime}$. Assume by contradiction that for some $s, t \in S$ we have $\neg(s<t)$ and $s<t$. By $(S$ to $<)$ we have that for all spheres $S$ such that $s \in S, t \in S$, Since $s<t$ we have that $s \in S_{t}$. Therefore we have that $t \in S_{t}$, which entails that $t<t$ against the irreflexivity of $<$. Contradiciton. For $<^{\prime} \subseteq<$, assume by contradiction that for $s, t$ in $S, \neg(s<t)$ and $s<^{\prime} t . B y(S$ to $<)$ we have that there exists $S_{k}$ such that $s \in S_{k}$ and $t \notin S_{k}$. Therefore we have that $s<k$ and $\neg(t<k)$. Therefore $s<t$. Contradiction.

We can now return to the main proof, where we established that there exists a sphere $T \in \mathbf{S}$, such that $T \cap \hat{a} \neq \emptyset$ and $s \notin T$. Let $t \in T \cap \hat{a}$. $B y(S$ to $<)$ we have that $t<^{\prime} s$. But since $s \in M_{<, a}$ we have that $\neg(t<s)$. This extracts a contradiction from assuming that $s \notin S_{m, a}$, but $s \in M_{<, a}$. In order to complete the proof that $S_{m, a}=M_{<, a}$ we can assume by contradiction that $s \in S_{m, a}$, but $s \notin M_{<, a}$. This proof proceeds along the same lines than the previous one.

DEFINITION 4.9. Let $M^{\prime}=\langle S, l, \mathbf{S}\rangle$ be the associated spheres model of a ranked model $M=\langle S, l,<\rangle$. We can then construct a core system of possible worlds corresponding to $M$ ', and a core model for $M$, as follows. Let $\langle W, \mathbf{C}, V\rangle$ be a core model for $M$, where $W=\{w \in U$ : there is $s \in S$ and $l(s)=w\}, \mathbf{C}=\{\{w:$ there is $s \in T$ and $w=l(s)\}: T \in \mathbf{S}\}$ and $V$ is the valuation used in probabilistic models (i.e. a function from $L$ to measurable events in a Boolean sub-algebra of the power set of $W$ ).

If $C_{m, a}$ is the minimal $\mathrm{V}(\mathrm{a})$ core, we can define here as well that $a \sim_{R} b$ is satisfied in the core model $\langle W, \mathbf{C}, V\rangle$ as long as every world $w$ in $V(a) \cap C_{m, a}$ is also a $b$-world.

Lehmann and Magidor point out in their paper that whenever a preferential model contains two states which bear the same label (i.e, the labelling function maps the two states to the same world), there might not exist an equivalent model with the same domain in which the

\footnotetext{
13 In fact, as we claimed above, ranked models and its associated sphere models can be correlated one-to-one. It is not difficult to show that if $\mathbf{S}$ is the system of spheres of a model $\mathrm{M}^{\prime}=\langle S, l, \mathbf{S}\rangle$ associated to a ranked model $\mathrm{M}=\langle S, l,<\rangle$, and $<^{\prime}$ is the relation extracted from $\mathbf{S}$ via $(\mathrm{S}$ to $<)$, then $\mathbf{S}=\left\{S_{x}: x \in S\right\} \cup \mathrm{S}$.
} 
labeling function is the identity function. So, states cannot be straightforwardly eliminated from ranked models. Notice, nevertheless, that given any ranked model $\mathrm{M}=\langle S, l,<\rangle$ the core system for its associated spheres model is such that for any assertion $a \sim_{R} b$ which is satisfied in $\mathrm{M}$, the assertion is also satisfied in $\langle W, \mathbf{C}, V\rangle$. Or to put it in a different way, for every ranked model there is a semantically equivalent core model with a different domain where the labeling function is the identity function. Here is a more formal statement of this fact;

LEMMA 4.5. A GHP is valid in an associated sphere model $M^{\prime}=$ $\langle S, l, \mathbf{S}\rangle$ if and only if it is valid in its corresponding core model $\langle W, \mathbf{C}, V\rangle$.

PROOF 4.3. Let $C_{m, a}$ be defined as $[a] \cap(\cap\{C \in \mathbf{C}: C \cap[a] \neq \emptyset\})$. Then the gist of the proof is to show that $S_{m, a} \subseteq \hat{b}$ if and only if $C_{m, a}$ $\subseteq V(b)$.

Assume by contradiction that $S_{m, a} \subseteq \hat{b}$, but that there is $w \in C_{m, a}$ and $w \notin V(b)$. Therefore there is $s \in S_{m, a}$, where $l(s)=w$. Since $s \subseteq \hat{b}$, $l(s)=w \in V(b)$. Contradiction.

Assume by contradiction that $C_{m, a} \subseteq V(b)$ but that there is $s \in S_{m, a}$ and $s \nsubseteq \hat{b}$. Now, notice that for every state $s$, if $s \in S_{m, a}$, then $l(s) \in$ $C_{m, a} \subseteq V(b)$. So, $s \in \hat{b}$. Contradiction.

THEOREM 4.1. If the underlying language is logically finite, $C A$ probabilistic models are complete with respect to $\mathcal{R}$ i.e. if a $\sim_{R} b$, then there is a CA probabilistic model $M$, such that $a \sim_{<M} b$.

PROOF 4.4. The gist of this proof is as follows. The canonical models used in (Lehmann and Magidor, 1990) in order to characterize $\mathcal{R}$ are rarely well-founded. But it the underlying language is finite, then all canonical models are well-founded. Therefore we know (via the completeness result stated in (Lehmann and Magidor, 1990)) that for any conditional assertion $a \sim_{R} b$ there is a well-founded ranked model $M$ $=\langle S, l,<\rangle$ such that $a \sim_{R} b$ is satisfied in M. Moreover, there is an associate spheres model for $M, N=\langle V, l, \mathbf{S}\rangle$, and a core model for $N$, $\langle W, \mathbf{C}, V\rangle=C$, both of which are semantically equivalent to $M$.

Construct now the probabilistic model $M^{\prime}=\langle\langle W, F\rangle, P, V\rangle$, where the universe of the probabilistic space $\langle W, F\rangle$ is the universe of the core model $C, V$ is the valuation used in $C$ and $P$ is a two-place function such that its core system is $\mathbf{C}$.

As a result of a previous lemma we have that the ranking orderings of $C A$ models are well-founded. So, the model $\langle\langle W, F\rangle, P, V\rangle$ is $C A$ and when the model is extended with $\sim_{<M^{\prime}}$, we have that $a \sim_{<M^{\prime}}$ b. 
When $L$ is countable, the situation is a little more involved. In this case $\mathcal{R}$ is no longer complete with respect to CA probabilistic models. The following example (based on a similar example, presented in Lemma 1 of (Lehmann and Magidor, 1990)) illustrates this point.

LEMMA 4.6. When $\mathrm{L}$ is countable, there is a rational relation that is defined by no CA probabilistic model.

PROOF 4.5. Let PL be the propositional calculus on the propositional letters $p_{i}, i \in N$ ( $N$ being the natural numbers). Define now $M=$ $\langle\langle U, F\rangle, P, V\rangle$ as follows:

$U=\left\{s_{i}: i \in N \cup\{w\}\right\}$

Now, let $P$ be any two-place function such that its induced ranking ordering $<_{M}$ is defined:

$s_{i}<_{M} s_{j}$ iff $i>j$

Let $s_{i} \in V\left(p_{j}\right)$ iff $j \geq i$, for $i \in N \cup\{w\}$ and $j \in N$. So, $M$ defines a rational relation such that for all $i \in N$, we have $p_{i} \sim_{<M} p_{i+1}, p_{i+1}$ $\sim_{<M} \neg p_{i}$ but $p_{0} \sim_{<M}$ false. Now, it is clear that any probabilistic model $M=\langle S, P, V\rangle$, inducing such a relation needs to contain an infinitely descending chain of points in the universe $U$ of $S$. Therefore the model $M$ is not $C A$.

So, a natural suggestion is to investigate probabilistic models where $\mathrm{CA}$ is not necessarily required. We will call such models finitely additive (FA).

LEMMA 4.7. If the underlying language contains countably many propositional letters, there is a FA probabilistic model $M$ such that, a $\sim_{P M}$ $b$ if $a \sim_{R} b$,

PROOF 4.6. Lehmann and Magidor prove in their paper (see section 5.4, theorem 10) a completeness result of $\mathcal{R}$ in terms of nonstandard probabilistic models. Basically if the language $L$ is countable (this assumption cannot be dispensed with):

$a \sim_{R} b$ iff $\operatorname{st}(p(B \mid A))=1$

where $p(. \mid$.$) is a non-standard measure and st(v) is the unique stan-$ dard number that differs from $v$ by at most an infinitesimal amount. Now it is known, by a result due to McGee (McGee, 1994), that if $p$ is a non-standard measure, and $p(A \mid U) \neq q 0$, then there is a FA two-place function such that:

$$
P(B \mid A)=\operatorname{st}(p(B \mid A))
$$

Therefore if $a \sim_{R}$ b there is a FA probabilistic model where a $\sim_{P M}$ $b$ is satisfied. So, a $\sim_{P M}$ b is complete with regard to a $\sim_{R}$ b. 
It is not difficult to see that a $\sim_{P M}$ b is also sound with respect to a $\sim_{R}$ b. Nevertheless, a $\sim_{<M}$ b fails to be sound with respect to a $\sim_{R}$ b. All the following results assume that the underlying language is countable.

LEMMA 4.8. When $M$ is finitely additive $\sim_{<M}$ is not sound with respect to $\sim_{R}$

PROOF 4.7. Take $U$ as before:

$U=\left\{s_{i}: i \in N \cup\{w\}\right\}$

and let $L$ contain a propositional letter $p$ such that:

$|p|=U-\left\{s_{w}\right\}$

(since $F$ is closed under differences $U-\left\{s_{w}\right\}$ is a measurable event in $F$ ).

Therefore, we have $p \sim_{<M} \neg p$, even when $p \uparrow_{R} \neg p$.

Nevertheless, soundness does hold with respect to the system $\mathcal{R} \mathcal{R}$ introduced in (Lehmann and Magidor, 1990).

LEMMA 4.9. When $M$ is a FA probabilistic model $\sim_{<M}$ is sound with respect to $\sim_{R R}$

DEFINITION 4.10. A probabilistic model $M=\langle S, P,|.|$.$\rangle is smooth if$ and only if for every sentence $\alpha$ its corresponding measurable event $|\alpha|$ is smooth with respect to the ranking ordering induced by $P$.

Now, it is clear that the relation $\sim_{P M}$, satisfied in all FA probabilistic models, is identical to the relation $\sim_{<M}$ induced by the sub-class of smooth and FA probabilistic models. In other words, when the language is infinite (but countable) $\mathcal{R}$ can be characterized either in terms of the relation $\sim_{P M}$ induced by FA models; or in terms of the relation $\sim_{<M}$ induced by the class of FA models which are smooth. ${ }^{14}$

Expectation acceptance tests can be characterized nicely in probabilistic terms. But, as we just showed, when the language is logically infinite, the characterization is in terms of finitely additive probabilistic models. In this paper we have focused on the countable case, i.e. when the universe is countable. Acceptance tests can be extended to the uncountable case, but as it is argued in (Arló-Costa, 2001) it is not clear that this extension preserves the main idea behind the use of Ramsey's bridges relating conditional probability and probability of conditionals.

\footnotetext{
${ }^{14}$ An account of conditional probability compatible with our model is the one offered by Lester Dubins in (Dubins, 1975). See (Seidenfeld, 2001) for an overview of the interest and limitations of finitely additive measures.
} 
When the models (and the language) are infinite, but countable, the Ramsey-Adams tests seem to offer a probabilistic articulation of the notion of expectation used in (Gärdenfors and Makinson, 1994) and (Gärdenfors, 1993) in order to characterize defeasible inference.

\section{Expectation test and epistemic paradox}

When the underlying language is logically finite and Countable Additivity (CA) is imposed we have a perfect alignment between acceptance of a rational conditional $a \sim_{P M} b$ and the fact that B is expected for $P$, given $A$. Using the terminology displayed in our Proof 4.1, $a \sim_{P M} b$ holds whenever $\operatorname{Ex}(P[A]) \subseteq B$.

Now, we saw that the situation is more involved when we increase the expressive power of the underlying language. In fact, when the language is infinite, but countable, we saw that there are very good reasons for abandoning CA. What can we say about the role of expectations in this case? To begin with we cannot define expectations as propositions including the innermost core, because such a core might not exist. An alternative definition can be used. The idea is to define the expectations for a function $P$ as follows:

$$
\operatorname{Exp}(P)=\{A: A \text { is entailed by some core of } P\} .
$$

As a matter of fact this is the spirit of the definition used by van Fraassen in (van Fraassen, 1995). When CA holds $P[A](B)=1$ guarantees that some core for $P[A]$ entails $B$. Once countable additivity is abandoned (as noted in (Arló-Costa, 2001)) it is possible that there is no core for $P[A]$ entailing $B$ although $P[A](B)=1$. In other words, $P[A](B)=1$ is only a necessary but not a sufficient condition for $B$ being expected given $A$ (for $P$ ).

As an example consider a universe $\Omega$ containing exactly the natural numbers $N$. Let $C_{0}=N$ and let $C_{i}$ be $N-S_{i}$ where $S_{i}$ is the final initial segment of the naturals of length $i$. This determines a system of cores in the space. Let $r_{n}=C_{n}-C_{n+1}$ be a rank for the system of cores (where '-' is set substraciton). Consider the probability function $P(. \mid \Omega)$ such that $P(B \mid \Omega)=1$ if $B$ is co-finite. For the remaining infinite but not co-finite events in the space, set $P(B \mid \Omega)$ to 1 if $\mathrm{B}$ is the proposition of the lowest rank among $\mathrm{B}$ and its complement, and set $P(B \mid \Omega)$ to 0 otherwise. Even when $P(O d d \mid \Omega)=1$, the event $O d d$ is not expected for the given system of cores (it is not entailed by any core $C_{i}$ ). This seems a good feature of cores. Assume that $P(B \mid \Omega)$ measures the likelihood that the outcome of an experiment (measured as a value in $N$ ) is in $B$. Then $P$ assigns a uniform zero probability to each exact value in $N$ 
$(P(\{w\} \mid \Omega)=0$, for every natural number $w \in N)$. And each co-finite set receives measure one.

Notice nevertheless that, counter to intuition, the agent represented by $P$ must assign measure one to the event stating that the value of the outcome is $O d d$. Moreover, independently of the particular definition of $P$, the agent should be certain that the outcome is odd or he should be certain that the outcome is even. Of course he should be certain that the outcome is either odd or even, but he need not be certain that it is odd or certain th at it is even, when the initial uniform prior is zero. Notice that the core system provided above (which is compatible with $P$ ) improves on this regard. In fact, even when $O d d$ has measure one, neither Odd nor Even are expected events (they are not entailed by any core for $P$ ).

\section{References}

Adams, E.: 1975, The logic of conditionals, Dordrecht: Reidel.

Arló-Costa, H. and Shapiro, S.: 1992, 'Maps between nonmonotonic and conditional logics,' Principles of Knowledge Representation and Reasoning: Proceedings of the Third International Conference eds. Nebel, B., Rich, C., Swar tout, W., 553-564.

Arló-Costa, H.: 1999, 'Qualitative and Probabilistic Models of Full Belief,' Proceedings of Logic Colloquim'98, Lecture Notes on Logic 13, S. Buss, P.Hajek, P. Pudlak (eds.), ASL, A. K. Peters.

Arló-Costa, H. and Thomason, R.: 2001, 'Iterative probability kinematics' Journal of Philosophical Logic 00, 46, 479-524.

Arló-Costa, H.: 2001, 'Bayesian Epistemology and Epistemic Conditionals: On the Status of the Export-Import Laws,' Journal of Philosophy, Vol. XCVIII, 11, 555-598.

Blume, L., Brandenburger, A., Dekel, E.: 1991, 'Lexicographic probabilities and choice under uncertainty,' Econometrica 59(1), 61-79.

Cross, C. and Nute, D.: 1998, 'Conditional Logic,' in Handbook of Philosophical Logic, second edition, Vol III Extensions of Classical Logic (eds.) D. Gabbay and F. Guenthner Reidel, Dordrecht.

De Finetti, B.: 1936, Les probabilités nulls Bulletins des Sciences Mathématiques (premiere partie) 60, 275-288.

Delgrande, J.: 1987, 'A first-order logic for prototypical properties,' Artificial Intelligence 33, 105-130.

Dubins, L.E.: 1975, 'Finitely additive conditional probabilities, conglomerability, and disintegrations,' Ann. Prob. 3:89-99.

Gabbay, D. M.:1985, 'Theoretical foundations for nonmonotonic reasoning in expert systems,' in Proceedings NATO Advanced Study Institute on Logics and Models of Concurrent Systems, (ed.) K.R. Apt, 439-457.

Gärdenfors, P.:1993, 'The role of expectations in reasoning,' Lund University Cognitive Studies, 21.

Gärdenfors, P., Makinson, D.:1994, 'Nonmonotonic inference based on expectations,' Artificial Intelligence 65:197-245. 
Ginsberg, M.: 1986, 'Counterfactuals,' Artificial Intelligence 30, 35-79.

Grove, A.: 1988, 'Two modelings for theory change,' Journal of Philosophical Logic, $17,157-170$.

Halpern, J.: 2000, 'Lexicographic probability, conditional probability and nonstandrad probability. Manuscript, Cornell University, November 14, 2000.

Hammond, P. J.: 1994, 'Elementary Non-Archimedean representations of probability for decision theory and games,' in Humphreys (ed.), Patrick Suppes: Scientific Philosopher; vol 1, Kluwer.

Hawthorne, J.: 1998, 'On the Logic of Nonmonotonic Conditionals and Conditional Probabilities: Predicate Logic,' Journal of Philosophical Logic, Volume 27, 1-34.

Jeffreys, H.: 1961, Theory of Probability, New York, Oxford, 3rd edition, 25.

Katsuno, H., Satoh, K.: 1996, 'A Unified View of Consequence Relation, Belief Revision and Conditional Logic' in Conditionals from philosophy to computer science. (eds.) G. Crocco, L. Fariñas del Cerro, H. Herzig, Oxford Univer sity Press, 33-65.

Kraus, S., Lehmann, D. and Magidor, M.:1990, 'Nonmonotonic reasoning, preferential models and cumulative logics,' 44 Artificial Intelligence, 167-207.

Kyburg, H. E., Jr.: 1961, Probability and the logic of rational belief, Wesleyan University Press. Middletown.

Lehmann, D., Magidor, M.: 1992, 'What does a conditional base entails?' Artificial Intelligence, $55,1-60$.

Levi, I.: 1988, 'Iteration of conditionals and the Ramsey test', Synthese, 76, 49-81.

Levi, I.: 1996, For the sake of the argument: Ramsey test conditionals, Inductive Inference, and Nonmonotonic reasoning, Cambridge University Press, Cambridge.

Lewis, D.: 1976, 'Propoabilities of conditionals and conditional probabilities', The Philosophical Review, 85, 297-315.

Makinson, D.:1989, 'General theory of cumulative inference,' in Proceedings Second International Workshop on Nonmonotonic Reasoning, Lecture Noters in Computer Science, (ed. M. Reinfrank), Springer Verlag, Berlin.

McGee, V.: 1994, 'Learning the impossible,' in Ellery Eells and Brian Skyrms (eds.) Probability and Conditionals: Belief Revision and Rational Decision, Cambridge University Press, Cambridge, England, 179-199.

Parikh, R. and Parnes, M.: 1972, 'Conditional Probability can be Defined for Arbitrary Pairs of Sets of Reals,' Advances in Math, 9, 520- 522.

Pearl, J.: 1988, Probabilistic Reasoning in Intelligent Systems: Networks of Plausible Inference, Morgan Kaufmann, Monterrey, California.

Popper, K.: 1968, The Logic of Scientific Discovery, Hutchinson, London, revised edition.

Renyi, A.: 1970, Foundations of Probability, San Francisco: Holden-Day.

Seidenfeld, T.: 2001, 'Remarks on the Theory of Conditional Probability: Some Issues of Finite versus Countable Additivity,' Probability Theory: Philosophy, Recent History and Relations to Science eds. V. F. Hendricks, S. A. Pedersen and K.F. Jorgensen, Kluwer Academic Publishers, Dordreccht, 167-178.

Shoham, Y.: 1987, Reasoning About Change, Cambridge, Mass., MIT Press.

Spohn, W.: 1988, 'A general non-probabilistic theory of inductive inference,' Causation in Decision, Belief Change and Statistics W. Harper and B. Skyrms (eds.) Dordrecht:Reidel,105-134.

van Fraassen, B.: 1995, 'Fine-grained opinion, probability, and the logic of full belief,' Journal of Philosophical Logic, 24, 349-377. 
coresnm.tex; 17/02/2004; 23:23; p.24 